\title{
Optical and digital processing II
}

\author{
David Casasent \\ Department of Electrical Engineering \\ Carnegie-Mellon University \\ Pittsburgh, Pennsylvania 15213
}

\section{Andrew G. Tescher}

The Aerospace Corporation

P. O. Box 92957

Los Angeles, California 90009

The second part of the joint special issue on optical and digital processing consists of thirteen papers. This second set of papers surveys recent optical and digital work in various specific data processing applications and techniques. The list of subjects addressed may not be comprehensive, but it is representative of the commonality of techniques and applications in the two powerful disciplines of optical and digital processing.

The paper by A. F. Gmitro, et al., summarizes the many algorithms and techniques utilizing optical processing in the reconstruction of images from their projections, as required in transaxial tomography.

Industrial application of computed tomography is discussed by R. P. Kruger, G. W. Wecksung and R. A. Morris. This paper primarily presents activities of Los Alamos Scientific Laboratory.

T. M. Cannon and E. E. Fenimore review the field of coded aperture imaging. This paper is applicable to both digital and optical processing methods.

In his paper, B. R. Frieden discusses an image restoration procedure. The concept of maximum information is introduced and utilized for restoration.

The paper by J. R. Fienup provides a discussion of an iterative digital processor approach to one image restoration problem. For additional work on iterative image processing, the reader is referred to the IOCC 1980 conference (part of the SPIE East, April 1980). An entire session was devoted to iterative image processing at this meeting.

The interaction of the human visual system with image coding is described by N. C. Griswold. The optimization of the coding procedure is based on psycho-physical considerations.
The number of signal processing applications relating to stereo imagery are limited. Thus, the paper by T. W. Ryan, R. T. Gray, and B. R. Hunt on application of the Cramer-Rao lower bound to correlation errors in stereo-pair images is of special interest.

The paper by W.T. Rhodes surveys recent progress in one aspect of incoherent optical processing, performing bipolar operations using novel system architectures and hybrid optical/digital techniques. (Alternate incoherent processing which is not covered here involves the use of LED sources, CCD shift registers and time integration.)

The paper by W. Frei, M. Singh, and T. Shibata describes digital change detection for image processing application. Their examples include remote sensing, radiology and nuclear medicine.

A novel optical processing approach described by J. F. Walkup is the fabrication and use of a space-variant optical processor.

The paper by R. P. Akins, R. A. Athale, and S. H. Lee surveys US, European and USSR research in optical feedback systems.

The paper by N. J. Berg, et al., discusses their recent progress on surface acoustic wave optical signal processors. The high frequency and bandwidth of advanced signal processors and the need for real-time processing of such data appear to be a realm in which acousto-optic and surface acoustic wave devices promise to make imminent breakthroughs and provide lasting impacts.

P. Kellman's paper is the first comprehensive survey of new optical signal processing architectural research where 1-D AO devices are used to realize 2-D processing functions. Time integrating, triple product and chirp-Z acousto-optic processors are described. These two papers provide an excellent updated survey of the state of the art in these two optical signal processing technologies. 